Appl. Phys. 10, 41-51 (1976)

\title{
Index Modulation and Spatial Harmonic Generation in Dichromated Gelatin Films
}

\author{
S. K. Case and R. Alferness \\ ECE Dept., The University of Michigan, Ann Arbor, MI 48104, USA \\ Received 2 October 1975/Accepted 5 January 1976
}

\begin{abstract}
A model for volume hologram formation in thick dichromated gelatin films is given. Using the model and a few basic film measurements, we can quantify the index modulation vs. exposure curve. The effects of saturation and dynamic absorption increase during exposure are considered in the generation of spatial harmonics. The effects of exposure with non-unity beam-ratio and multiple incoherent exposures are predicted and experimentally verified. The thick transmission grating diffraction efficiencies resulting from first- and higher-order index modulations are calculated and compared to experiments. The methods and conclusions are applicable to other phase materials.
\end{abstract}

Index Codes: $42.65,42.30$

Hardened dichromated gelatin is a holographic phase material that is essentially lossless after development and can be made to yield volume holograms of very high index modulation. It is the purpose of this paper to develop a model for dichromated gelatin which, together with a minimum number of measured parameters, will quantify the dependence of index modulation on exposure under various exposure conditions. Because some of the film properties we report can also be seen in tests of other materials, it is expected that many of the predicted effects and our analytic methods will apply in general to a large class of holographic films.

The model presented is both a mathematical and physical one. The form of the equations chosen to represent various film quantities is based on our macroscopic observation that the absorption vs. exposure curve and the first-order index modulation vs. exposure curve both have the form of saturating exponential functions (which saturate at different rates depending on the film preparation conditions). It is these functional forms that prompt us to give a mathematical treatment to the numerical values of the index curves. Results of past $[1,2]$ and present investigations, and knowledge of the chemistry of dichromated colloids also lead us to postulate as to the origin of the index variation in these films.

\section{Chemistry and Hologram Formation Mechanism}

The chemistry of the photo-induced hardening process in dichromated gelatin films was treated extensively by Kosar [3] and is shown in Figure 1. Briefly, a gelatin film is sensitized in an ammonium dichromate solution and then allowed to dry. During exposure, light is absorbed to reduce the $\mathrm{Cr}^{+6}$ ion to $\mathrm{Cr}^{+3}$ which forms several intermediate chromium compounds. During development, the chromic ion, $\mathrm{Cr}^{+3}$, acts to form a crosslink bond between the carboxylate groups of neighboring gelatin strands. This bond gives rigidity (hardness) to the gelatin.

For hologram formation, the exposure of the film is not uniform, as in a sinusoidal exposure resulting from the interference of two incident plane waves (Fig. 2). Highly exposed and relatively unexposed regions will be hardened to different degrees during development, causing periodic strain within the gelatin. When the firm is rapidly dehydrated with isopropanol 


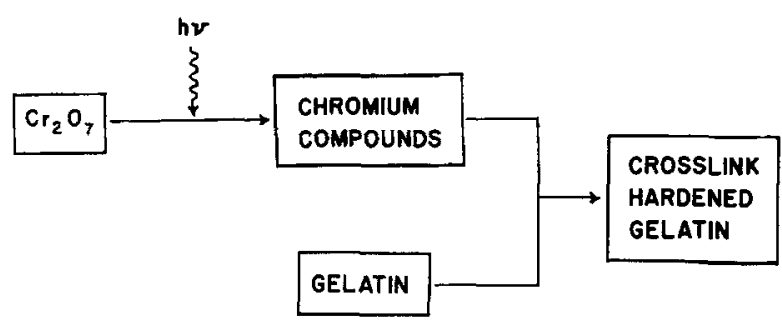

Fig. 1. The photo-induced crosslink bond

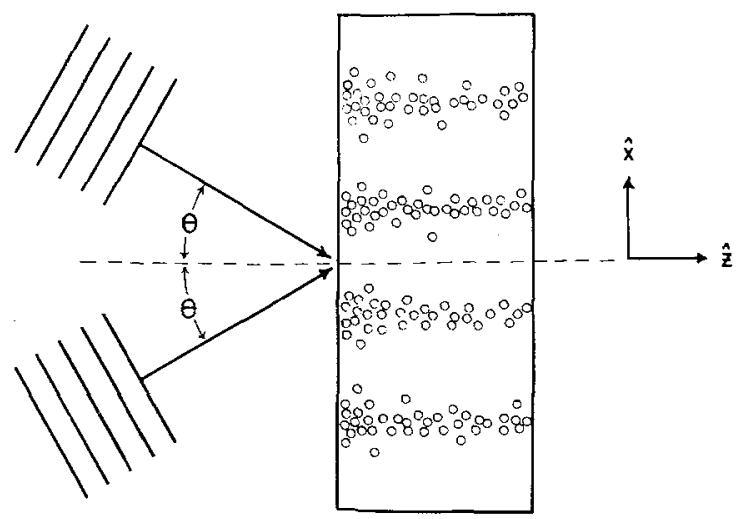

Fig. 2. Two plane waves expose the film. The sinusoidal exposure causes differential hardening resulting in the formation of small vacules in the gelatin. The fringes are assumed to be normal to the film surface

during the last step of development, the strain is increased and then thought to be relieved by the formation of fissures or cracks in the vicinity of the unhardened gelatin chains [1]. We picture the fissures as enlarged separations between the gelatin strands comprising the emulsion [4]. We can represent the enlarged separations as small vacuoles with varying number density within the volume of the gelatin as in Figure 2. The local dielectric constant is consequently reduced in the area containing a mixture of gelatin and vacuoles. Extensive experiments by Curran and Shankoff with index matching fluids that wet gelatin indicate that the presence of voids within the gelatin is quite reasonable [1]. They have suggested that these air gaps are large in size and few in number (one gap/exposure fringe). We propose an extension of this model by assuming that the voids are instead small in size and large in number. This idea was mentioned by Curran and Shankoff and is consistent with nearly all of their experimental observations for transmission holograms, as will be discussed later. A model including a large number of small vacuoles (dimensions $\ll \lambda$ ) in a gelatin matrix would account for the low scatter noise observed with this film and would allow for a smooth average index variation across an exposure fringe. As we shall see, a smooth index variation is consistent with experiment in that it would allow for the sinusoidal index variation necessary for our observed diffraction efficiencies and angular selectivities, and would produce much less higher-order diffraction at low exposures than would the square wave index modulation [1] associated with a single crack/fringe.

For small vacuoles embedded within a dielectric medium, the change in the local dielectric constant is given by the Lorentz-Lorenz formula $[5,6]$ which for small modulations becomes

$\varepsilon(x) / \varepsilon_{0}=1+c^{\prime} \sum N(x) \alpha_{p}(x)$.

The local dielectric constant at a position $x$ depends on the product of the number density $N(x)$, and the polarizability $\alpha_{p}(x)$ of the vacuoles. (The polarizability is proportional to the volume, shape, and relative dielectric constant of the vacuole $[6,7]$.) The local index of refraction is then $n(x)=\sqrt{\varepsilon(x)}$.

\section{The Model}

To model the film response as a function of exposure, we now consider and quantify the dynamic absorption increase of the sensitized film during exposure, the photo-induced hardening of the film, and the resultant index modulation. Following this, we relate firstand second-order index modulation to diffraction efficiency at the first- and second-order Bragg angles and compare our predicted results with experiments.

\subsection{Dynamic Absorption}

It is well known that during exposure, the sensitized dichromated gelatin film slowly changes from a bright orange-yellow to a darker color, with an accompanying increase in absorption. The photoinduced color change and absorption increase is due to the formation of the intermediate chromium compounds in Figure 1 [3]. Thus, we can relate the absorption to the number density of exposed chromate complexes, $\varrho_{E}$. We write the absorption coefficient as $\alpha=\alpha_{1}+\beta \varrho_{E}$,

where $\alpha_{1}$ is a bulk absorption (due to dark reaction, total dichromate concentration, etc.). Because $\varrho_{E}$ 
increases with exposure $E$, we write in general the differential equation

$d \varrho_{E}=k \varrho_{u} d E$,

where $k$ depends on the quantum efficiency of the exposure reaction and $\varrho_{u}$ is the density (number/ volume) of unexposed chromates. For an absorbing material, the exposure will decrease with depth in the emulsion, so that by integrating (3), we get

$\varrho_{E}(z)=\varrho_{0}\left[1-\exp \left(-k E e^{-\int_{0}^{z} \alpha d z^{\prime}}\right)\right]$

where $E=I_{0} t$ and $\varrho_{0}=\varrho_{u}+\varrho_{E}$. This is correct if $\alpha$ is a very slowly varying function of $\varrho_{E}$. In general, since the absorption coefficient varies considerably with exposure and hence with depth, we must integrate numerically to find $\varrho_{E}(z, E)$ and $\alpha(z, E)$.

\subsection{Film Hardening and Index Modulation}

Because we can measure the absorption vs. exposure, we can find $\varrho_{E}$ in terms of macroscopic observables. Since it is the exposed chromates that initiate crosslink bonds ultimately resulting in hologram formation, it is convenient to express the term $\sum N \alpha_{p}$ in (1) in terms of $\varrho_{E}$. (For low exposures, $\varrho_{E}$ is linearly related to exposure and we could use the two interchangably.) We start by observing the first-order diffraction efficiency $\mathrm{DE}_{1}$ for sinusoidal exposures of the form

$E=E_{0}(1+m \cos \kappa x)$.

where $\kappa=2 \pi f$, and $f$ is the grating frequency.

From the $\mathrm{DE}_{1}(E)$ curve and the $\varrho_{E}(E)$ curve, we can obtain the first-order index modulation $n_{1}(E)$ or $n_{1}\left(\varrho_{E}\right)[8]$ (see Fig. 5).

With knowledge of the shape of the index curves and of film hardening properties, we can postulate as to the microscopic origin of the index modulation. Because vacuole formation is thought to result from the stress associated with differential hardening of the film, we assume the term $\sum N \alpha_{p}$ to be proportional to the differential hardness $H=H(\max )-H(x)$ across an exposure fringe. (The proportionality factor would contain any spatial frequency response - although we observe a uniform response from $800-1200 \mathrm{l} / \mathrm{mm}$.) Since the film hardness is related to the number of crosslink bonds and the first bonds have more effect on film hardness than later bonds [9], we assume a form for the hardness

$H=H_{0}\left[1-\exp \left(-k_{H} \varrho_{E}\right)\right]$, where $H_{0}$ will depend on the dichromate concentration and preparation conditions. Inserting the above into (1), we obtain

$\frac{\varepsilon(x)}{\varepsilon_{0}}=1-C H_{0}\left[\left(1-e^{-k_{H} \varrho_{E}(\max )}\right)-\left(1-e^{-k_{H} \varrho_{E}(x)}\right)\right]$,

where the constant $C$ is a positive number and the minus sign preceeding it reflects the negative relative polarizability of a vacuole. For an exposure of the form of (5), (7) will give an index distribution which can be expanded in the form

$n=\sum_{q} n_{q} \cos q \kappa x$.

For small exposures, only $n_{0}$ and $n_{1}$ will be appreciable and we can expand the exponentials in (4) and (7) to obtain the depth averaged coefficients (for film thickness $D$ )

$$
n_{0}=\varepsilon_{0}^{\frac{1}{2}}\left[1-\Omega_{1} m E_{0}\right]
$$

and

$$
n_{1}=\varepsilon_{0}^{\frac{1}{2}} \Omega_{1} m E_{0}
$$

with

$\Omega_{1}=\frac{1}{2} C H_{0} k_{H} k \varrho_{0}\left[\frac{1-\exp \left(-\alpha_{1} D\right)}{\alpha_{1} D}\right]$.

It should be emphasized that although we gave physical arguments leading up to (7), it is our experimental observation that the first-order index modulation curve has the form of a saturating exponential function that initially lead us to the form of (7). We note that the $\varrho_{E}(\max )$ term is a bias term that assures that there are no vacuoles at the maximum exposure position on a fringe (where the film is hardened the most). We also note that the form of (7) correctly predicts that when the film receives a large, spatially uniform bias exposure and then a small modulated exposure, that

$$
\frac{\varepsilon(x)}{\varepsilon_{0}}=1-C H_{0} k_{H} e^{-k_{H} \varrho_{E}(\mathrm{BIAS})}\left[\varrho_{E}^{\prime}(\max )-\varrho_{E}^{\prime}(\mathrm{x})\right] .
$$

That is, the spatially uniform response (bonding) from the bias exposure causes an exponential decrease in sensitivity to the modulated exposure. This response has been observed experimentally [12].

Thus from our observations, we arrive at a simple form for the modulation of the dielectric and hence index of refraction. 


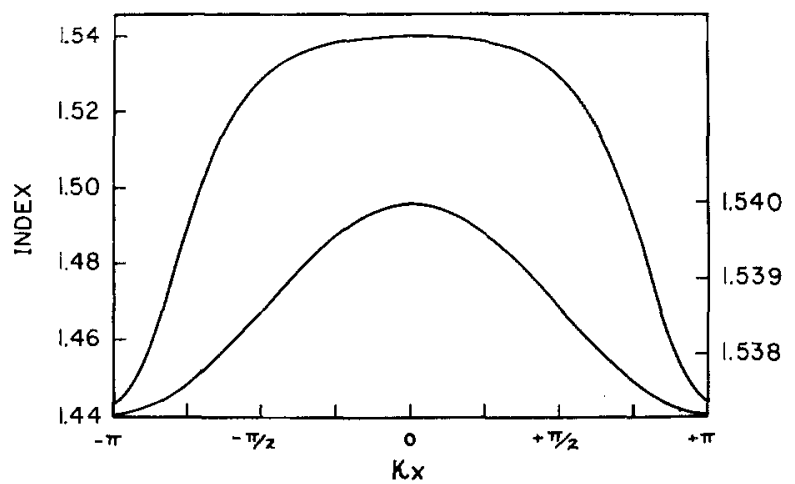

Fig. 3. Index of refraction vs. position across one fringe. Upper curve (left scale) is a high exposure $\left(E_{0}=650 \mathrm{~mJ} / \mathrm{cm}^{2}\right)$; lower curve (right scale) is a low exposure $\left(E_{0}=4 \mathrm{~mJ} / \mathrm{cm}^{2}\right)$

\subsection{Spatial Harmonic Generation}

Because the form of the total index curve (7) was based on our observations of the shape of the first-order index modulation curve, we will expect good numerical agreement between predicted and measured values of $n_{1}(E)$. A better test of our model is to see how well it predicts higher-order index modulations.

Our model includes two mechanisms for the generation of spatial harmonics: absorption distortion and hardness saturation. In absorption distortion, the absorption increase in highly exposed areas near the front surface of the emulsion shields the deeper regions of the emulsion (for that same $x$ position) from exposure. This effect is greatest at the fringe maxima and occurs to a lesser degree in lower exposure areas across the fringe. The resulting exposure for the deeper layers of the emulsion is distorted and is no longer a single frequency sinusoid, resulting in harmonic generation.

Hardness saturation also occurs most rapidly at the exposure maxima causing a decreased ability to respond to further exposure at these positions. For high exposure levels, the resulting differential sensitivity at different positions across a fringe causes a nonlinear response to increased exposure, which results in harmonic generation.

We can graphically see the results of the combined effects if we look at the computed shape (see next section for computation method) of one fringe at different exposure levels. For low level, sinusoidal exposures, where we have a linear response, the lower curve in Figure 3 shows a sinusoidal index variation as a function of position. For high exposures, the upper curve in Figure 3 shows a flat-top index profile caused by saturation and absorption distortion. The upper curve, of course, will have a great deal of harmonic content and considerable higher order diffraction.

\subsection{The Computer Model}

Because of dynamic absorption, saturation effects, and sinusoidally varying exposures, the values of $\varrho_{E}, H$, and hence $n(E)$ vary with depth, exposure and transverse position. In order to accurately compute the index $n(x, z)$, we have written a computer program for the following steps. Beginning with the preexposure initial conditions $\varrho_{E}(x, z, E=0)=0$, and $\alpha=\alpha_{1}$, we write the lowest exposure $E_{1}=\bar{E}_{1}(1+\cos \kappa x)$. Dividing the thick emulsion into a series of thin slabs $(\Delta z=0.5 \mu \mathrm{m})$ in the $z$ direction and narrow channels in the $x$ direction $\left(\Delta x=1 / 32\right.$ fringe), we insert $E_{1}$ into (4) and compute $\varrho_{E}\left(x, z=0.5 \mu \mathrm{m}, E_{1}\right)$ for all positions $x$ over one interference fringe. Using these values and (7), we compute $n\left(x, z=0.5 \mu \mathrm{m}, E_{1}\right)$ and then expand it into its $x$ dependent frequency components (8). We repeat this process for all $z$ planes and then obtain the averages over $z$

$n_{0}=\frac{1}{D} \sum n_{0}\left(z, E_{1}\right) \Delta z$

$n_{1}=\frac{1}{D} \sum n_{1}\left(z, E_{1}\right) \Delta z \ldots$

We next increase the exposure by a small amount to $E_{2}$ and use the previous values of $\varrho_{E}\left(x, z, E_{1}\right)$ with (2) to compute the absorption coefficient for use in (4). The entire computation is continued to find $n_{0}\left(E_{2}\right)$, $n_{1}\left(E_{2}\right), n_{2}\left(E_{2}\right)$, etc.

\subsection{Diffraction Efficiency}

We cannot measure $n_{1}$ and $n_{2}$ directly to compare with our model but instead must measure diffraction efficiency (DE) and relate this to the spatial index modulation. At the first-order Bragg angle, we have [8]

$\mathrm{DE}_{1}=\sin ^{2}\left(\frac{\pi n_{1} D}{\lambda \cos \theta}\right)$,

where $n_{1}$ is averaged in depth [13] as in (10) and (12) and $\lambda$ is the free-space wavelength.

For readout at the second-order Bragg angle, ( $2 \sin \theta_{2 B}=2 \lambda f / n_{0}$ ), both single diffraction from the harmonic grating $n_{2}$, and double diffraction from the fundamental grating $n_{1}$ contribute to the $\mathrm{DE}$ so that 
in general, an equation of the form of (13) is not adequate. A three wave analysis has shown [14]

$$
\begin{aligned}
\mathrm{DE}_{2}= & \frac{1}{4}\left\{\left[\cos \chi-\cos \left(\frac{3}{2} \gamma_{2}+\xi\right)\right]^{2}\right. \\
& \left.+\left[\frac{\Delta}{\chi} \sin \chi-\sin \left(\frac{3}{2} \gamma_{2}+\xi\right)\right]^{2}\right\},
\end{aligned}
$$

where

$$
\begin{gathered}
\gamma_{1}=\frac{\pi n_{1} D}{\lambda \sqrt{\cos \theta_{2 B}}}, \quad \gamma_{2}=\frac{\pi n_{2} D}{\lambda \cos \theta_{2 B}}, \\
\xi=\frac{\pi \lambda f^{2} D}{n_{0}\left(1+\cos \theta_{2 B}\right)}, \quad \Delta=\xi-\frac{\gamma_{2}}{2}
\end{gathered}
$$

and

$$
\chi=\sqrt{4^{2}+2 \gamma_{1}^{2}} \text {. }
$$

The frequency dependence (see the $\xi$ term) is due to the double diffraction mechanism. For the high exposure levels required to obtain appreciable $\mathrm{DE}_{2}$, three output waves are observed. Because some of the energy goes into a first order, $\mathrm{DE}_{2}$ cannot be made arbitrarily to reach $100 \%$ (see Fig. 8) [14].

\subsection{Initial Parameters}

Our last remaining task is to determine the parameters for our various equations. We have two saturating exponential equations (absorption and index) and an intitial absorption coefficient, so that five independent parameters must be determined. From measurements of the slope and intercept of the low exposure absorption curve with

$\bar{\alpha}=\alpha_{1}+\beta \varrho_{0} k\left[\frac{1-\exp \left(-\alpha_{1} D\right)}{\alpha_{1} D}\right]$

and the saturation level absorption $\bar{\alpha}=\alpha_{1}+\beta \varrho_{0}$, we can find three parameters. These can be used with the measurements of $n_{1}(E)$ for low exposures (10 and 13) and for the saturation value of $n_{1}(E)$ to find the two remaining parameters.

\section{Experimental Investigation}

We are now ready to check our mathematical model with our experiments. The dichromated gelatin films used in this investigation were prepared from Kodak $649-\mathrm{F}$ plates $[15,16]$. Unless otherwise noted, a $5 \%$ ammonium dichromate solution was used as the sensitizer and the film was exposed at $\lambda=4880 \AA$

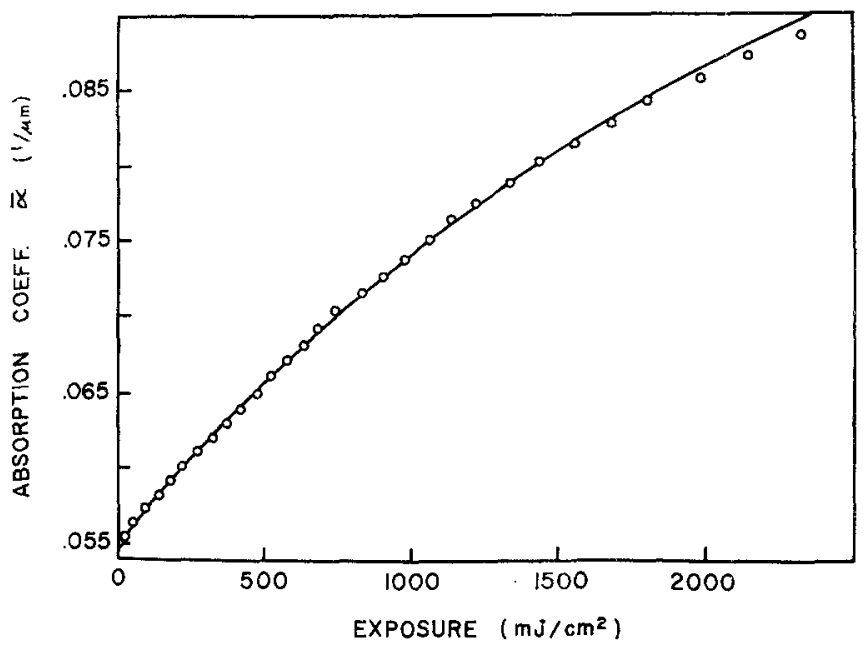

Fig. 4. Average absorption coefficient as a function of exposure (for $5 \%$ sensitizer solution)

with a spatial frequency of $1180 \mathrm{l} / \mathrm{mm}$. We have assumed the emulsion thickness to be $16 \mu \mathrm{m}$, a number obtained from angular selectivity measurements of the developed gratings.

\subsection{Absorption Tests}

Using a $5 \%$ sensitized plate, we measured the transmittance of the film as a function of exposure and used this data to find $\bar{\alpha}$ vs. E. Figure 4 shows the measured and computed values of $\bar{\alpha}$ in the exposure range of interest for hologram formation. The intial value of $\bar{\alpha}$ shows that only $42 \%$ of the incident light reaches the back layers of the emulsion. At high exposure levels, $25 \%$ of the light is transmitted. The absorption can be decreased by using a lower sensitizer concentration or a longer exposure wavelength $[17$, $18]$.

\subsection{Index Modulation}

On the same plates, we record plane wave gratings with $100 \%$ modulation $(m=1)$. From measurements of DE vs. exposure, we use (13) to find $n_{1}(E)$. Inserting our measured parameters into our computer program, we compare the measured and predicted first-order index modulation in Figure 5. The data points are taken from five different plates and thus represent the reproducibility of the process. We notice that over the lower two decades of exposure, the film responds quite linearly and then gradually decreases in sensitivity as the film begins to saturate. 


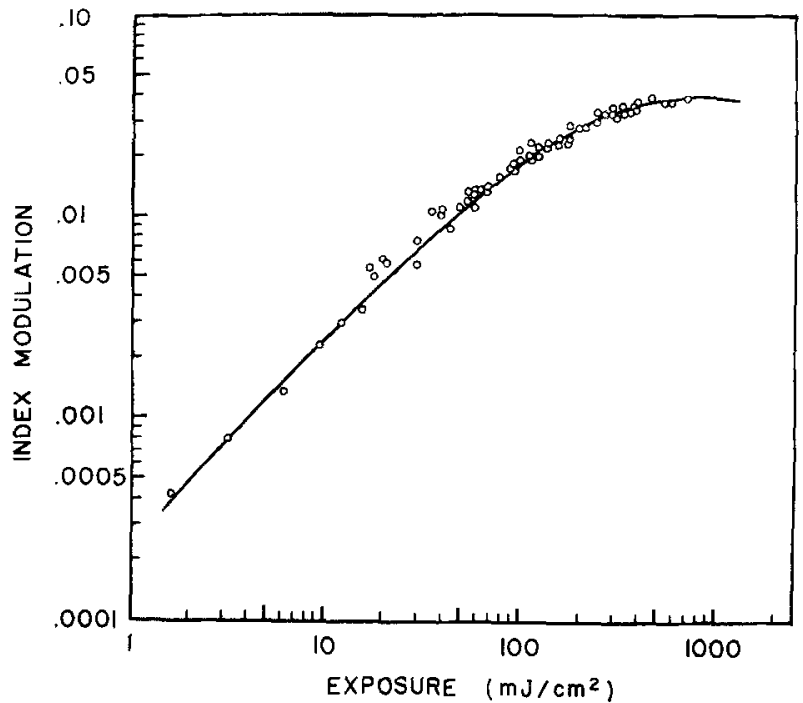

Fig. 5. First order index modulation vs. exposure for $5 \%$ sensitizer. Theory (-); Data (O)

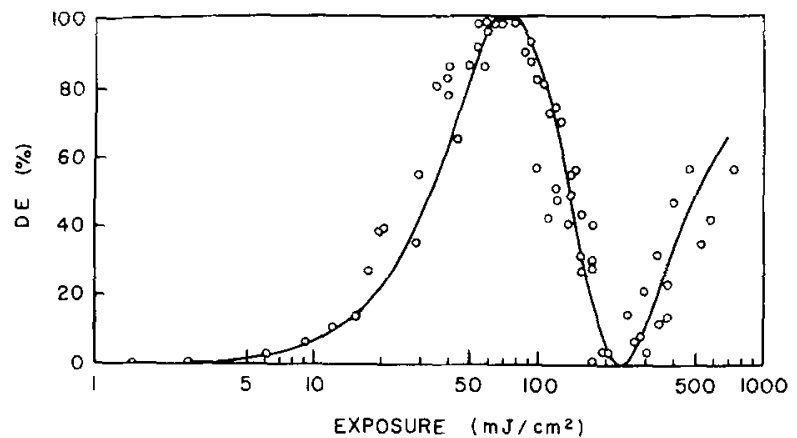

Fig. 6. Diffraction efficiency vs. exposure for plane wave gratings using $5 \%$ sensitizer. Theory (-); Data (O)

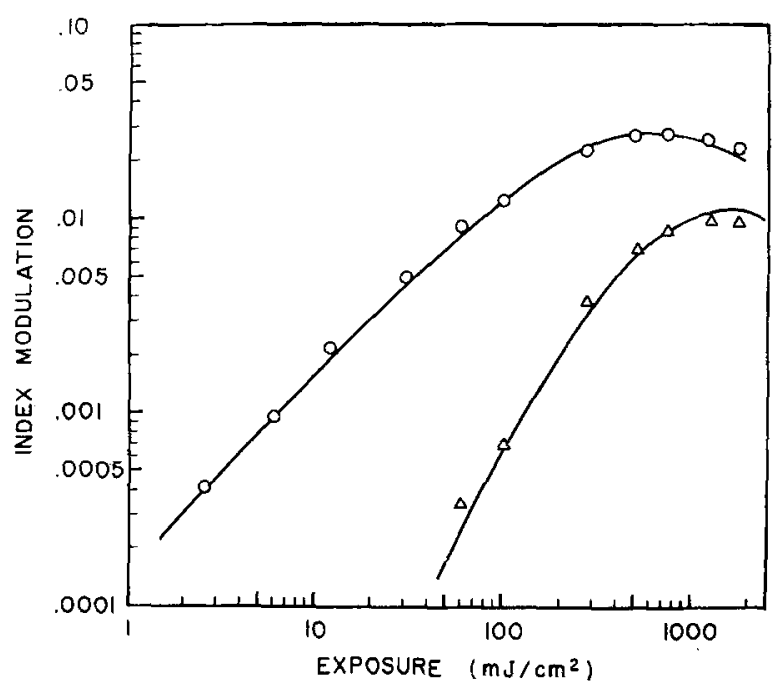

Fig. 7. First order index modulation $\left(O=n_{1}\right)$ and second-order index modulation $\left(A=-n_{2}\right)$ vs. exposure for $1 \frac{1}{2} \%$ sensitizer
In Figure 6, we show the computed and measured first-order $\mathrm{DE}$ resulting from the index modulations in Figure 5. The DE has been corrected for surface reflection losses. There is a negligible ammount of absorption after development so that nearly $100 \%$ of the incident energy can be diffracted into the first-order. Conjugate and higher orders for read out at the first-order Bragg angle contain at most 2\% of the energy for our particular exposure conditions $\left(Q=2 \pi \lambda f^{2} D / n_{0}=45\right)[8]$.

\subsection{Spatial Harmonics}

To test our model for harmonic generation, we look at data from a plate sensitized with $1 \frac{1}{2} \%$ ammonium dichromate solution and exposed with plane waves at $f=860 \mathrm{l} / \mathrm{mm}$. At this sensitizer concentration (compared to $5 \%$ ), the absorption coefficient is considerably reduced and the gratings are of nearly uniform modulation (in depth, z). This is desirable because (14) was derived assuming uniform index modulation. (In fact, observations of the minima of the first-order angular selectivity curves $[13,19]$ for low and high exposure gratings indicate that the modulation gradient does not become greatly exaggerated at high exposure levels. This may be because the first-order index modulation in the front layers begins to saturate while that in the back layers continues to increase until the modulation becomes more nearly uniform.)

For read out of the gratings at the first and then the second-order Bragg angles, followed by the use of (13) and (14), we can find $n_{1}(E)$ and $n_{2}(E)$. Using the computer model, we can also compute the values of $n_{1}(\mathrm{E})$ and $n_{2}(E)$. Figure 7 shows the good agreement between theory (solid line) and experimental data points. We note that $n_{1 \max }$ for the $1 \frac{1}{2} \%$ sensitizer has a lower value than that for the $5 \%$ sensitizer. As expected, $n_{2}$ begins to have appreciable values in the exposure region where $n_{1}$ begins to deviate from linearity. At high exposures, $n_{2}$ contributes appreciably to the total index modulation $\left(\left|n_{2}\right|=0.5 n_{1}\right)$.

By keeping terms in $\varrho_{E}^{2}$ for a low exposure expansion of the index, we find the form $n_{2}=-\Omega_{2} E^{2}$. From the slope of the $n_{2}$ curve (Fig. 7), we see the $E^{2}$ dependence and from the inversion of $\mathrm{DE}_{2}$ data with (14) we see that $n_{2}$ is negative relative to $n_{1}$.

Figure 8 shows the good agreement between the measured DE's for this plate and the DE computed using the solid lines in Figure 7 and (13) and (14). The position and magnitude of the peak $\left(\mathrm{DE}_{2}\right)$ in the 


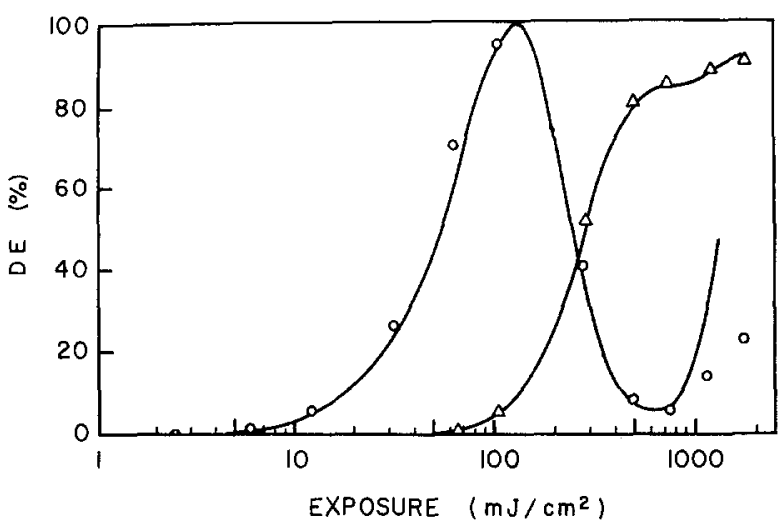

Fig. 8. Measured diffraction efficiency at the first-order Bragg angle $(O)$ and at the second-order Bragg angle $(\Delta)$. Theory $(-)$

second order differs with sensitizer level because the generation of $n_{2}$ is related to the saturation of $n(E)$ and the absorption distortion, both of which change with sensitizer level. From observations of a number of gratings and computer study, we find that saturation is the most important consideration in spatial harmonic generation with absorption distortion providing a smaller contribution $(\approx 15 \%)$. Because saturation is the dominant effect and the $1 \frac{1}{2} \%$ film saturates at a lower value of index modulation (see Figs. 5 and 7), we find that for a given value of $n_{1}$, the $1 \frac{1}{2} \%$ film will have a larger value of $n_{2}$ than will the $5 \%$ film. As a result, $\mathrm{DE}_{2}$ peaks earlier (relative to the $\mathrm{DE}_{1}$ curve) for the lower sensitizer concentration films because saturation effects come into play at lower modulation levels. (However, it should be remembered that the maximum index modulation is higher for the $5 \%$ film and thus at high exposure levels it will achieve larger values of both $n_{1}$ and $n_{2}$.)

We have also observed third-order generation in these films and have measured $70 \% \mathrm{DE}$ at the third-order Bragg angle for high exposure $\left(E=2300 \mathrm{~mJ} / \mathrm{cm}^{2}\right)$. The DE equation at the third-order Bragg angle would be considerably more complex than (14) and in general it is easier to compute the DE numerically [20]. If the spatial frequency is sufficiently high and the emulsion sufficiently thick so that only two orders are significant, the analysis of Su and Gaylord can be used [21].

One of the most striking features of Figure 8 is that the first-order DE peaks near $100 \%$ and then decreases for increasing index modulation [as predicted by (13)] but does not come down to zero (near $E=650$ ) before starting to increase again. A very careful study of the angular selectivity for the gratings with
$E>650$ indicates that their index modulation is less than that of gratings recorded at $E=650 \mathrm{~mJ} / \mathrm{cm}^{2}$. We also notice that $n_{1}(E)$ in Figure 7 increases monotonically until $E \approx 650$ and then decreases as is evident in both the theory and the data. A decrease in modulation with increased exposure (an effect similar to solarization in silver based materials) occurs at very high exposure levels. We can understand this by referring to the shape of one fringe in Figure 3. After a certain exposure, the effective hardness at $k x=0$ will have saturated. Further exposure can still increase the hardness in the areas near the minimum exposure position $(\kappa x= \pm \pi)$ however. The net result is to make the hardness modulation components (and the resultant index modulation) more nonlinear. Because $n_{1}(E)$ is just the first term in the Fourier series expansion of $n(x, E)$, as $n(x, E)$ begins to saturate and look more like a square wave (with altered duty cycle), the high frequency components of modulation will increase while the low frequency terms will maximize (saturate) and for sufficiently high exposures, begin to decrease. Thus, an index modulation decrease with increased exposure is possible even under optimum exposure conditions. This effect occurs for materials other than dichromated gelatin and can even be seen in photoresists (a relief material) [15]. It should be noted that a decrease in modulation with increased exposure can also be caused by a slow, uniform fringe drift during exposure, or reduced fringe contrast during exposure (due to high frequency vibrations, beamratio [15] or pathlength difference), although in our experiments we believe this not to be the case.

\subsection{Beam-Ratio}

Up to this point, we have assumed that the incident plane waves used to expose the film were of equal irradiance. If the waves are of unequal irradiance, then the modulation $m$, see (5), is reduced. In Figure 9, we show the first-order index modulation curve for a $5 \%$ sensitized plate exposed with beam-ratio $=\mathrm{BR}=4$ (lower curve) and the corresponding $\mathrm{BR}=1$ curve from Figure 5 (upper curve). For $B R=4$, our modulation is reduced to $m=0.8$ and we see that in the linear region (Fig. 9), our predicted and observed index modulation is reduced by the same factor. In the lower portion of the index curve, if we desire a given index modulation $n_{1}$, and a higher $\mathrm{BR}$ (to take advantage of the greater $S / N$ available at higher BR [15]), we can compensate for the reduced fringe 


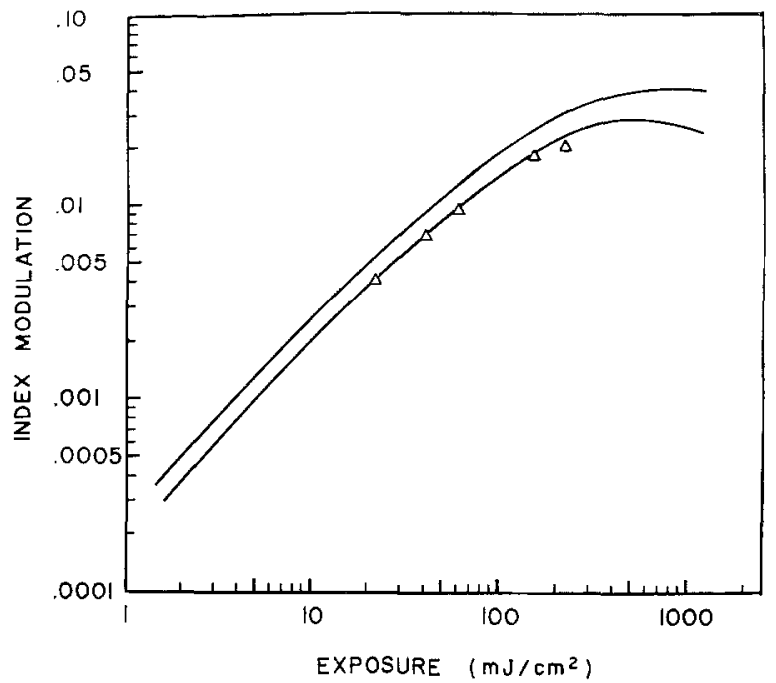

Fig. 9. Comparison of first-order index modulation for $\mathrm{BR}=1$ (upper curve) and $\mathrm{BR}=4$ (lower curve)

modulation by increasing the total exposure. Saturation effects will, of course, lower the maximum available index modulation.

The effect of index modulation maximization and then decrease with increased exposure is quite evident in the high BR experiments. In dichromated gelatin data reported by Colburn et al. [15] with $\mathrm{BR}=50$, the DE (and corresponding index modulation) is seen to peak near $25 \%$ and then decrease for increasing exposure.

\subsection{Multiple Exposures}

In earlier works $[20,22,23]$, we have investigated the diffraction effects of propagation through a thick material that has been multiply exposed. For that investigation, we assumed that the film had a sinusoidal first-order index modulation for each of two gratings $\left(n_{1-1}\right.$ and $\left.n_{1-2}\right)$ and proceeded to predict the diffraction efficiencies resulting from these overlapping modulations. In the present investigation, we wish to find the effect of multiple exposures on the film itself so that we can predict the first-order modulations $n_{1-1}$ and $n_{1-2}$ as a function of exposure. We can then combine the effects of film properties and wave propagation to relate the DE to exposure under multiple exposure conditions.

We have shown that a way to avoid interaction between two gratings exposed on one film is to angularly separate the gratings during exposure such that their Bragg angles are far removed from one another [23]. Under these conditions, we observe that each of the two gratings diffracts as if it were a single exposure grating. Thus, for far separated gratings, we can to good approximation apply (13) to relate the index modulation of each grating to its corresponding DE.

In order to predict the effect of multiple incoherent exposures on the film, we first look at the case where the film receives a sinusoidal first exposure and then a second exposure that has uniform spatial intensity

$E=E_{1}(1+\cos \kappa x)+E_{2}$.

Inserting this into (4 8), we see that for low exposures,

$n_{1-1}=\Omega_{1} m E_{1}$

which is the same as (10). Thus in the region where the film responds linearly, there is no effect on the first exposure due to the second exposure. For higher exposures, where the film saturation becomes important, the "bias build-up" associated with higher overall exposure will inhibit cracking and will reduce the modulation $n_{1-1}$.

We now consider two sinusoidal exposures with far removed Bragg angles. Since we compute the modulation at successive $z=$ constant planes, we write

$$
\begin{aligned}
E= & E_{1}\left[1+\cos \left(\kappa_{1} x+\phi_{1}(z)\right)\right] \\
& +E_{2}\left[1+\cos \left(\kappa_{2} x+\phi_{2}(z)\right)\right],
\end{aligned}
$$

where $\phi_{1}(z)$ and $\phi_{2}(z)$ describe the changing overlap of the two two sets of fringes with distance $z$. In the low exposure, linear response region, insertion of (18) into (4-8) will again show that the index modulation of $n_{1-1}$ of the first exposure is unchanged by the presence of the second. For equal exposure times, the index modulation of the second exposure, $n_{1-2}$, is slightly less than $n_{1-1}$ both because the film absorption increase due to the first exposure acts to effectively reduce the second exposure, and because the film is closer to saturation for the second exposure.

For higher exposure levels, the problem becomes more complex in that saturation effects are dependent on the exact location of the fringes in any $z=$ constant plane. In general, for high exposures, we can let our computer program keep track of the exposure densities $\varrho_{E 1}$ and $\varrho_{E 2}$ and then compute $n_{1-1}$ and $n_{1-2}$. For a thick emulsion and large angular offset fringes (so that the fringe patterns cross many times), if we follow the fringes $n_{1-1}$ through the emulsion, we will average $\cos \left(\kappa_{2} x+\phi_{2}(z)\right)$ through many cycles so that the effect of the second exposure is equivalent to the uniform bias of (16). 
In Figure 10, we show the predicted first-order index modulation per grating for a double exposure film (lower curve) with equal exposures for each grating. The upper curve is the single exposure curve from Figure 5 for reference. The triangles in Figure 10 represent the measured index modulation [obtained from DE data and (13)] of a double exposure film that received a sinusoidal first exposure and then a uniform second exposure. The circles represent the index modulations of a double exposure film that received two sinusoidal exposures with large angular separation. The upper and lower circles at a given exposure represent the first and second exposures. Clearly, we can see that for low exposures, where the film is in the linear region, each exposure responds as if it were the only exposure on the film. For higher exposures, we begin to see the effects of saturation. In order to reach a given index modulation in each of the two gratings, we will now have to expose each grating at a higher level than we would to make an equivalent single grating reach that modulation level. Of course, this compensation can only be carried out over a limited range because we see that the index modulation/grating saturates at a lower level in the double exposure case. In fact, each of the two gratings has a maximum modulation approximately equal to half of the maximum modulation of a corresponding single grating.

In Figure 11, we compare the predicted and measured DE for each of the two gratings on the double exposure film. The predicted DE is the average of the first and second exposures obtained by using the index curve from Figure 10 and (13) modified to this construction geometry. We note empirically that the maximum value of $\mathrm{DE}$ for each of the two decoupled gratings is near $100 \%$ and that the exposure/grating to reach this level is approximately twice that required for a single exposure grating to reach $100 \% \mathrm{DE}$. The exact compensation required, of course, depends on the index modulation level we are trying to reach. If we could modify the response properties of the film to extend the linear portion of the curve (by changing the sensitizer concentration, exposure wavelength, absorption properties, etc.) we might be able to improve both the maximum index modulation and the compensation required for multiple exposures [24].

We have also made triple exposures at far removed Bragg angles. The results at low and high exposures are similar to those for double exposures. We achieved a saturation level $\mathrm{DE}$ of approximately $75 \%$ for each

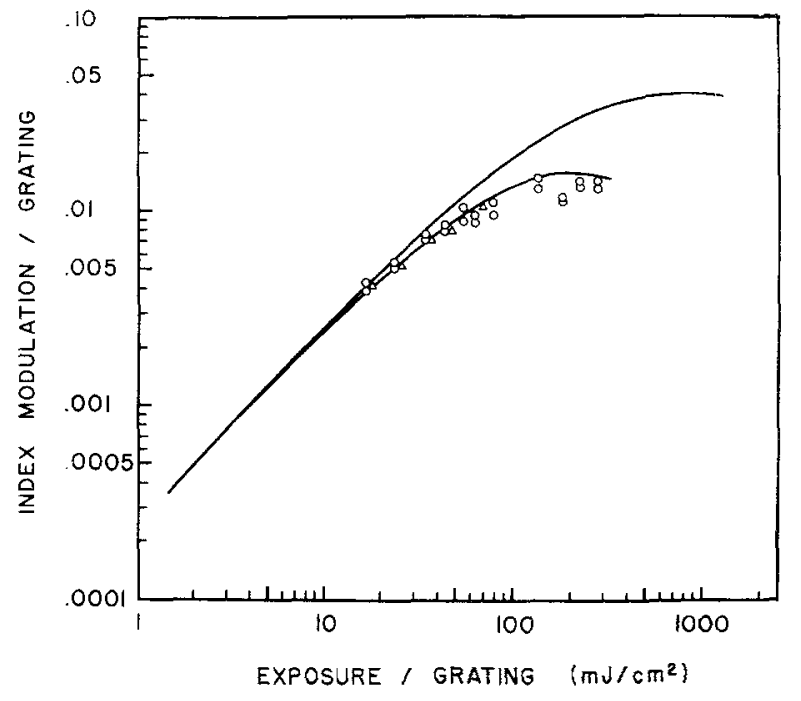

Fig. 10. Comparison of single exposure (upper curve) and double exposure (lower curve) index modulation/grating vs. exposure/ grating. Data points are from double exposure gratings; sinusoidal exposure + uniform exposure $(\Delta)$; two incoherent sinusoidal exposures $(0)$

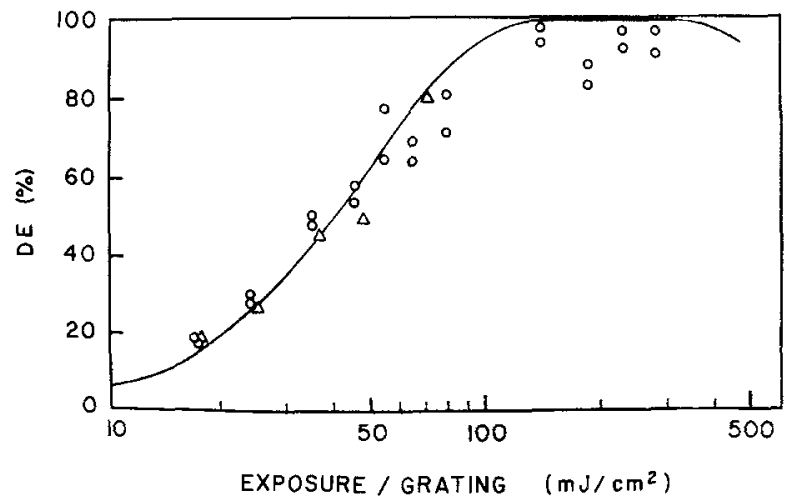

Fig. 11. Diffraction efficiency/grating as a function of exposure/ grating for uncoupled double exposures. Theory $(-)$

of the three decoupled gratings (at an exposure/ grating $\approx 220 \mathrm{~mJ} / \mathrm{cm}^{2}$ ). The maximum modulation/ grating is roughly $1 / 3$ that of an equivalent single grating.

\section{Discussion}

\subsection{Bulk Index}

The chief objection to the physical basis of this model (by previous investigators [1]) is that the mixture of gelatin and air vacuoles would lower the average index of refraction of the film. To see if a change in the bulk 


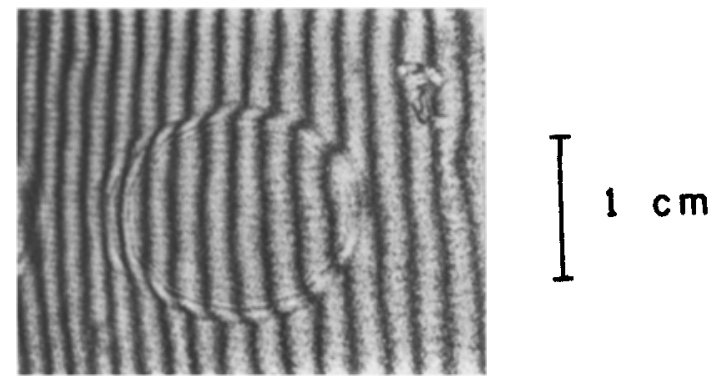

Fig. 12. Interferogram showing the decrease in bulk index in the exposed (circular) region

index is realistic, we placed a grating in a MachZehnder interferometer to observe any bulk phase shift between an exposed and the unexposed region. To compensate for thickness variations between exposed and unexposed portions of the gelatin [2], we cemented a flat cover plate over the gelatin surface using an optical cement that was index matched to the unexposed gelatin. In Figure 12, we see the interferogram of an exposed circle containing a $1000 \mathrm{l} / \mathrm{mm}$ plane wave transmission grating. The grating has slightly less than $100 \%$ DE (although we are not at the Bragg angle for this test). Since the decrease in bulk index in this exposure range is approximately equal to the amplitude of the index modulation $n_{1}$, see $(9$ and 10), we predict that the total phase difference between the exposed and unexposed region should be slightly less than $\frac{1}{2}$ wave, which is what we observe. As we interferometrically observed the gratings from which we obtained the data for Figure 5, we saw that for the lowest exposures, very little phase shift occurred while the highest exposures had a shift greater than one fringe. The fringe shift was in the direction indicating a decrease in the bulk index, in agreement with our model.

\subsection{Sensitivity of Results to the Model}

As was pointed our earlier, our model is based on experimental observations of the shape of the absorption and first-order index curves. From this we argued how differential hardening of the emulsion could lead to the observed index modulation. If the index modulation were the result of some other mechanism (e.g. the bonding of isopropal alcohol to the exposed chromate sites $[25,26]$ ) or a combination of several mechanisms, then one could deduce a functional form equivalent to our $H$ that yielded a mathematical form for the index modulation that is similar to ours. Thus, it is the mathematical form of the index curve that should be preserved.

The variables involved in our model are consistent with our experimental observations. For example, $n_{1}$ (for a given exposure) is observed to increase with sensitizer concentration as indicated in the form of (11). The bulk absorption coefficient follows a similar trend. Our function $H$, which we call the hardness, may not be an exact representation of the hardness of the film. In general, film hardness should be represented by a higher rank tensor since the planar orientation of the individual gelatin strands comprising the emulsion [10] would allow much larger cracks (e.g. layer splitting [1]) for holograms formed with fringes aligned with the gelatin fibers (reflection holograms). Furthermore, the observations that thin sheets of gelatin swell proportionately more than thick sheets [27] indicates greater stressing for thinner emulsions (and possibly large voids). Pre-hardening dichromated gelatin films, preparation temperatures, age of the sensitizer solutions and relative humidity will also have an effect on hologram formation and the index modulation $[10,11,16,18,28]$. All of these observations lead us to believe that a completely general treatment of the exact mechanism for hologram formation in dichromated gelatin films would be quite intricate. We have shown, however, that a fairly simple mathematical approach to the formation of transmission holograms, using variables that are reasonable in a physical and mathematical sense, predicts results that are in good agreement with experiment. Through the use of the model, we are able to quantify linear and nonlinear film response, effects of non-unity beam-ratio, index decrease at high exposures, and modulation properties of multiple exposures in thick films. By quantifying the dynamic absorption increase with exposure and the saturation of the $n_{1}(E)$ curve, we can use the model to predict the generation of harmonic-order index modulations which can be an important tool in studying the shape of fringes and thereby the modulation mechanism of a material.

Finally, we note that the response afforded by a microscopic index variation mechanism is consistent with our observations of fundamental and harmonic order diffraction. Observations of angular selectivity curves also indicate that our calculations of modulation gradients within the depth of the film are accurate and that the entire volume of the film contributes to the diffraction. 
Acknowledgements. We wish to thank Professors E. N. Leith and J. Upatnieks and Mr. B. Hansche for useful discussions throught the course of the work. Helpful discussions with B. J. Chang and W. S. Colburn are also acknowledged. This work performed under the support of NSF Grants GK-43148 and GK-42361.

\section{References}

1. R.K. Curran, T.A.Shankoff: Appl. Opt. 9, 1651 (1970)

2. L. H.Lin: Appl. Opt. 8, 963 (1969)

3. J. Kosar: Light Sensitive Systems (John Wiley, New York 1965) Ch. 2

4. This would be consistent with the webbing observed in photomicrographs of low frequency transmission gratings in [1]

5. M. Chang, N. George: Appl. Opt. 9, 713 (1970)

6. M. Born, E.Wolf: Principles of Optics, 2 nd. ed. (Macmillan, New York 1964) p. 87

7. S.B.Cohn: In Proc. of Symposium on Modern Advances in Microwave Techniques (Polytechnic Press, New York 1954) p. 472

8. H. Kogelnik: Bell Syst. Tech. J. 48, 2909 (1969)

9. Very low hardener concentrations are quite effective in hardening films. See [3,10 and 11]

10. J.E. Jolley: Photo. Sci. Eng. 14, 169 (1970)
11. C.E.K.Mees, T.H.James: The Theory of the Photographic Process (Macmillan, New York 1966) Ch. 3

12. G. Fillmore, R.Tynan: J. Opt. Soc. Am. 61, 199 (1971)

13. D. Kermisch: J. Opt. Soc. Am. 59, 1409 (1969)

14. R.Alferness: J. Opt. Soc. Am. 66 (1976) in press

15. W.S.Colburn, R.G.Zech, L.M.Ralston: Holographic Optical Elements, Tech. Rep. AFAL-TR-72-409 (Jan. 1973) p. 93

16. M.Chang: Appl. Opt. 10, 2550 (1971)

17. D.H.Close, A.Graube: Materials for Holographic Optical Elements, Tech. Rep. AFML-TR-73-267 (Oct. 1973) p. 32

18. B. J.Chang: Private communication

19. N. Uchida: J. Opt. Soc. Am. 63, 280 (1973)

20. R. Alferness: Appl. Phys. 7, 29 (1975)

21. S.F.Su, T.K.Gaylord: J. Opt. Soc. Am. 65, 59 (1975)

22. S. K. Case: J. Opt. Soc. Am. 65, 724 (1975)

23. R.Alferness, S. K. Case: J. Opt. Soc. Am. 65, 730 (1975)

24. For very thick emulsions $(D=100 \mu \mathrm{m})$, we have achieved higher average index modulations by using a $1 \frac{1}{2} \%$ sensitizer (with its lower absorption) than with use of a $5 \%$ sensitizer

25. D. Meyerhofer: RCA Review 33, 110 (March, 1972)

26. A. Graube: Private communication

27. J.Alexander: Glue and Gelatin (Chem. Catalog Co., New York 1923) Ch. 5

28. E. Bradbury, C. Martin: Proc. Roy. Soc. A214, 183 (1952) 